\title{
Dust and Molecule Formation and Processing in Supernovae and their Remnants
}

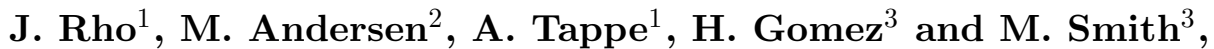 \\ J. P. Bernard ${ }^{4}$, T. Onaka ${ }^{5}$ and J. Cami ${ }^{6}$ \\ ${ }^{1}$ SETI Institute, 189 Bernardo Ave, Mountain View, CA 94043; \\ email:jrho@seti.org \\ ${ }^{2}$ Institut de Planétologie et d'Astrophysique de Grenoble, France \\ ${ }^{3}$ School of Physics and Astronomy, Cardiff University, The Parade, Cardiff, CF24 3AA, UK, \\ ${ }^{4}$ Centre d'Etude Spatiale des Rayonnements, CNRS, 9 av. du Colonel Roche, BP 4346, 31028 \\ Toulouse, France ${ }^{5}$ Department of Astronomy, The University of Tokyo, 7-3-1 Hongo, \\ Bunkyo-ku, Tokyo 113-0033, Japan, onaka@astron.s.u-tokyo.ac.jp, \\ ${ }^{6}$ Dept. of Physics \& Astronomy, Univ. of Western Ontario, London, ON N6A 3K7, Canada
}

\begin{abstract}
Supernovae (SNe) produce, fragment and destroy dust, molecules and nucleosynthetic elements, and reshape and modify the ISM. I will review recent infrared observations of supernova remnants (SNRs) and SNe which show that SNe are important sites of dust and molecule formation and are major dust creators in the Universe. Detection of carbon monoxide (CO) fundamental band from the young SNR Cas A indicates that astrochemical processes in SNRs interacting with molecular clouds provide astrophysical laboratories to study evolution of the ISM returning material from dense clouds into the more diffuse medium and galactic halo. Two dozen SNRs are known to be interacting with molecular clouds using $\mathrm{H}_{2}$ and millimeter observations. Recent Spitzer, Herschel and SOFIA observations along with ground-based observations have greatly advanced our understanding shock processing and astrochemistry of dust, $\mathrm{H}_{2}$, high J CO, and other neutral and ionized molecules and polycyclic aromatic hydrocarbon (PAH). Ionized molecules and warm layer of molecules that are excited by UV radiation, X-rays, or cosmic rays will be described. Finally I will discuss how astrochemical processes of dust and molecules in SNRs impact the large scale structures in the ISM.
\end{abstract}

Keywords. molecules, supernova remnants, Cas A, ISM, dust, infrared, molecular hydrogen

\section{Introduction}

Supernovae (SNe) play a key role in the chemical and dust budget of galaxies producing heavy elements and dust in their ejecta and processing dust via strong shocks both in the local and in the early Universe. SN explosions light up regions of stellar birth, trigger further star formation, return processed material to the ISM, providing the elements necessary for life. Recent evidence also suggests core-collapse SNe are dust factories, a dominant source of dust grains in galaxies throughout cosmic history.

Huge quantities of dust observed in high-redshift galaxies raise the fundamental question of the origin of dust in the Universe, as the low-intermediate mass stars that had been believed to be the primary source of dust were not evolved enough in high-redshift galaxies. In contrast, SNe could dominate the dust and molecule production (Nozawa et al. 2003; Todini \& Ferrara 2001; Cherchneff 2008) significantly altering the the star formation properties of early galaxies as compared to the Population III stars that first cause reionization. Evidence for this was scarce until recently. Our Spitzer and Herschel observations of young nearby SN remnants (SNRs) demonstrated, for the first time, 

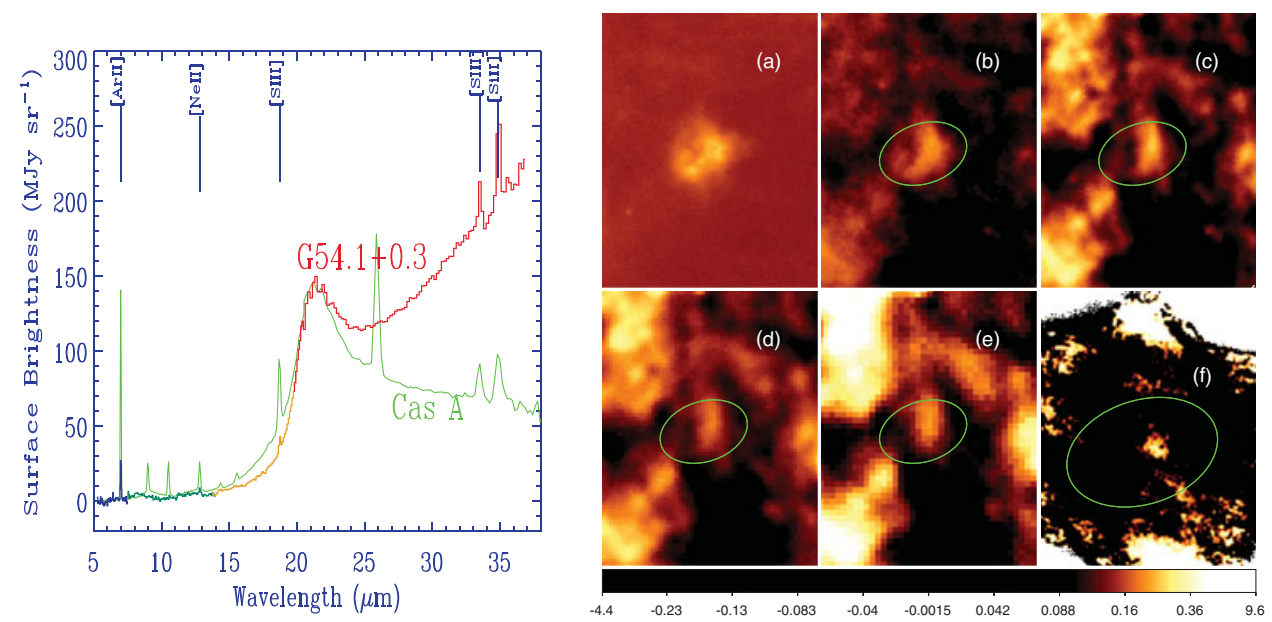

Figure 1. (a: left) Spitzer IRS spectrum of G54.1+0.3 shows strong $21 \mu \mathrm{m}$ dust feature with ionic lines of [Ar II], [Ne II], [S III], [Si II], and [S II]. (b: right) Cold dust of G54.1+0.3; Herschel PACS at 70 (a) and $160 \mu \mathrm{m}$ (b) and SPIRE at 250 (c), 350 (d) and 500 (e) $\mu \mathrm{m}$ and CSO 350 $\mu \mathrm{m}$ SHARCII (f) images. The size of the entire SNR is marked as an ellipse.

significant amounts of dust in SN ejecta, orders of magnitude more than previously detected (Rho et al. 2008; 2009a; Barlow et al. 2010; Gomez et al. 2012). These results imply that SNe could be responsible for the large dust masses detected in high redshift galaxies and in galaxies today, but only a handful of such observations exist.

We serendipitously discovered a dust feature peaking at $21 \mu \mathrm{m}$ from G54.1+0.3, and the dust feature as shown in Figure 1a is remarkably similar to that of Cas A from Rho et al. (2008). Strong correlation between 21- $\mu \mathrm{m}$ dust and Ar ejecta has been observed in Cas A. Ar ejecta is detected from the IRS spectra of G54.1+0.3 and IRAC $8 \mu \mathrm{m}$ map shows shell-like morphology indicating the distribution of Ar ejecta. We present detection of cold dust from G54.1+0.3 using CSO SHARCII $350 \mu \mathrm{m}$ map and Herschel PACS and SPIRE maps as shown in Figure 1b. G54.1 was observed with SHARC II on 2006 April 18 and 2009 May 3 and 4. SHARC II is a $12 \times 32$ bolometer array with field of view of $2.6^{\prime} \times 0.97^{\prime}$, observing at $350 \mu \mathrm{m}$ on the Caltech Submillimetre Observatory in Hawaii. The weather conditions were good during both runs, with $\tau_{225}$ ranging from 0.04 to 0.05 . Twenty-two scans were observed in total using the boxscan format scanning with on-source integration time of 3.7 hours. We identified cold dust from G54.1+0.3 using HIGAL survey (PI, S. Molinari) on the Herschel Space Telescope. In Figure 1b, the PACS 70 and $160 \mu \mathrm{m}$ and SPIRE 250 and $350 \mu \mathrm{m}$ images show clear detection of G54.1+0.3 and a shell-like morphology, that is similar to the MIPS $24 \mu \mathrm{m}$ map (Temim et al. 2010). The SPIRE map at $500 \mu \mathrm{m}$ also detected the SNR; however, that is somewhat confused with the background emission. The SHARCII image with a higher $\left(8^{\prime \prime}\right)$ spatial resolution than the Herschel images has detected the brightest part of the SNR in the Herschel $350 \mu \mathrm{m}$ map, showing consistent detection between CSO and Herschel. The cold dust detection from G54.1+0.3 will be one of a few cases from SNRs.

Our current understanding of molecule and dust chemistry and composition is limited. The current models assume that very little carbon is locked up in CO and almost all of carbon is available for dust formation. As a tracer of gas properties and dust formation, CO is potentially an important diagnostic for any supernova. We detected nearinfrared $2.29 \mu \mathrm{m}$ first overtone CO emission from Cas A with the Wide field InfraRed Camera (WIRC) on the Hale 200 inch telescope at Mount Palomar (Rho et al. 2009b). 
Subsequently we have spectroscopically confirmed the presence of the fundamental band at $4.5 \mu \mathrm{m}$ using the AKARI IRC spectrograph as shown in Figure 2a (Rho et al. 2012).

SNRs interacting with molecular clouds (MCs) provide critical laboratories to study shock physics, grain destruction, metal enrichment, and gas heating and cooling in dense interstellar gas. The most significant coolants of the shocked gas are predicted to be finestructure and metastable lines of atoms and ions, as well as $\mathrm{H}_{2}$ line emission (Hollenbach \& McKee 1989). We used archival Spitzer data to systematically search for IR counterparts of all 96 known SNRs covered by the GLIMPSE/IRAC Legacy survey (Reach et al. 2006), and many IRAC-detected SNRs reveal an interaction with dense gas through a $4.5 \mu \mathrm{m}$ excess, which indicates $\mathrm{H}_{2}$ and $\mathrm{CO}$ line emission. We also performed follow-up observations with IRS $(5-40 \mu \mathrm{m})$ and detected rotational $\mathrm{H}_{2}(\mathrm{~S}(0)$ to $\mathrm{S}(7))$ lines and rich IR ionic lines including O, N, S, Fe, and Ne (Hewitt et al. 2009; Andersen et al. 2011). The dust model fitting of the SEDs is done using the method described in Bernard et al. (2008) and the updated version from Compiégne et al. (2008). Three dust components are adopted, PAH molecules, Very Small Grains (VSG) and Big Grains (BG). The abundance of each dust species $\left(\mathrm{Y}_{P A H}, \mathrm{Y}_{V S G}, \mathrm{Y}_{B G}\right)$ and the strength of the radiation field $\left(\mathrm{X}_{I S R F}\right)$ are taken as free parameters in the fit. The fit results are shown in Figure $2 \mathrm{~b}$ (Andersen et al. 2011), showing that abundances of PAHs and VSGs are higher than those in the Milky Way and the LMC (Bernard et al. 2008). Typically the radiation field is 10-100 times larger than normal interstellar radiation ISRF, the strength of which is consistent with being created from the shock.

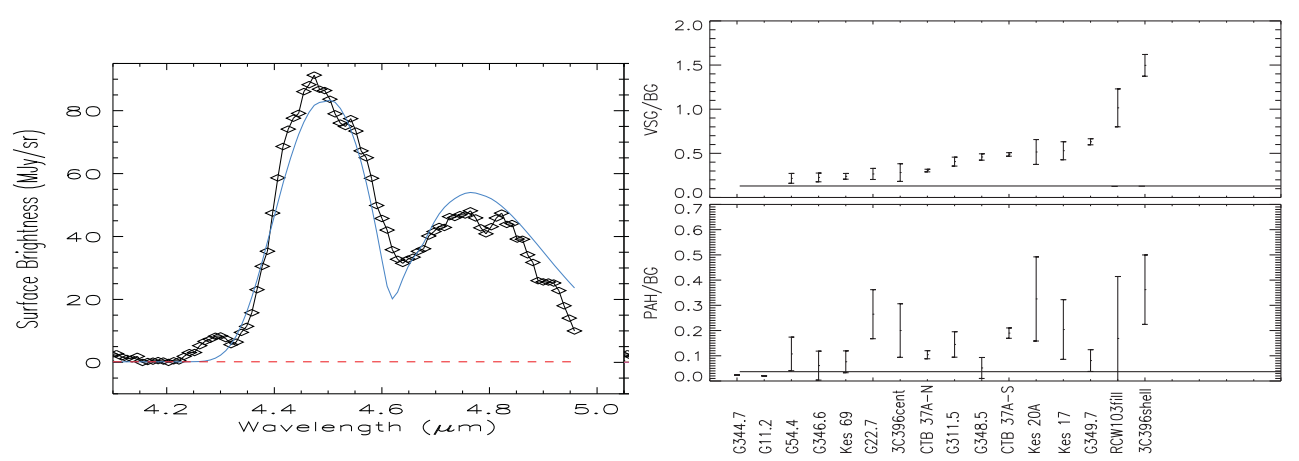

Figure 2. (a: left) High resolution grism spectrum (black) towards the southern part of Cas A. Superposed is the best fit CO model (blue) using a single component (left; velocity of $-4800 \mathrm{~km}$ $\mathrm{s}^{-1}$ ), suggesting that the $\mathrm{CO}$ may be at a location different from the ejecta in this case.

(b: right) The ratios of PAHs and very small grains to big grains are higher than those of the Milky Way (solid line) except two young SNRs.

\section{References}

Andersen, M., Rho, J., Reach, W. T., et al. 2011, ApJ 742, 7

Barlow, M. J., Krause, O., Swinyard, B. M., et al. 2010, A\& $A$ 518, L138

Bernard, J.-P., Reach, W. T., Paradis, D., et al. 2008, AJ 136, 919

Compiègne, M., Abergel, A., Verstraete, L., \& Habart, E. 2008, A\& A 491, 797

Hewitt, J. W., Rho, J., Andersen, M., \& Reach, W. T. 2009, ApJ 694, 1266

Hollenbach, D. \& McKee, C. F. 1989, ApJ 342, 306

Reach, W. T., Rho, J., Tappe, A., et al. 2006, AJ 131, 1479

Rho, J., Jarrett, T. H., Reach, W. T., et al. 2009, ApJ (Letters) 693, L39

Rho, J., Kozasa, T., Reach, W. T., et al. 2008, ApJ 673, 271

Rho, J., Onaka, T., Cami, J., \& Reach, W. T. 2012, ApJ (Letters) 747, L6

Rho, J., Reach, W. T., Tappe, A., et al. 2009, ApJ 700, 579

Temim, T., Slane, P., Reynolds, S. P., et al. 2010, ApJ 710, 309 